\title{
Ultrashort pulse CPA-free Ho:YLF linear amplifier
}

Moritz Hinkelmann, Dieter Wandt, Uwe Morgner, Jörg Neumann, Dietmar Kracht

Moritz Hinkelmann, Dieter Wandt, Uwe Morgner, Jörg Neumann, Dietmar Kracht, "Ultrashort pulse CPA-free Ho:YLF linear amplifier," Proc. SPIE 10511, Solid State Lasers XXVII: Technology and Devices, 1051109 (15 February 2018); doi: 10.1117/12.2287387

SPIE. Event: SPIE LASE, 2018, San Francisco, California, United States 


\title{
Ultrashort pulse CPA-free Ho:YLF linear amplifier
}

\author{
Moritz Hinkelmann ${ }^{\mathrm{a}}$, Dieter Wandt ${ }^{\mathrm{a}}$, Uwe Morgner ${ }^{\mathrm{a}, \mathrm{b}}$, Jörg Neumann ${ }^{\mathrm{a}}$, and Dietmar Kracht ${ }^{\mathrm{a}}$ \\ ${ }^{a}$ Laser Zentrum Hannover e.V., Laser Development Department, Ultrafast Photonics Group, \\ Hollerithallee 8, D-30419 Hannover, Germany \\ b'Institut für Quantenoptik, Leibniz Universität Hannover, Welfengarten 1, D-30167 Hannover, \\ Germany
}

\begin{abstract}
We present CPA-free linear amplification of $6.3 \mathrm{ps}$ pulses in Ho:YLF crystals up to $100 \mu \mathrm{J}$ pulse energy at $10 \mathrm{kHz}$ repetition rate. The seed pulses at a wavelength of $2.05 \mu \mathrm{m}$ are provided by a Ho-based all-fiber system consisting of a soliton oscillator and a subsequent pre-amplifier followed by a free-space AOM as pulse-picker. Considering the achieved pulse peak power at MW-level, this system is a powerful tool for efficient pumping of parametric amplifiers addressing the highly demanded mid-IR spectral region. In detailed numerical simulations we verified our experimental results and discuss scaling options for pulse duration and energy.
\end{abstract}

Keywords: Ultrafast lasers, solid-state lasers, laser amplifiers, infrared lasers, ultrashort lasers, mode-locked lasers

\section{INTRODUCTION}

Ultrafast high-energy laser sources operating in the spectral region around $2 \mu \mathrm{m}$ are of great interest due to a variety of applications in medicine, communication, micromaching of polymers and metrology. ${ }^{1}$ Additionally, they can be used as pump source for the generation of mid-infrared (mid-IR) radiation in optical parametric amplifiers (OPAs). ${ }^{2}$ Especially, by using non-oxide crystals such as ZGP with its transparency window between $2 \mu \mathrm{m}$ to $8 \mu \mathrm{m}$ in those amplifiers, high conversion efficiency would be achieved. ${ }^{3}$ Common types of laser sources in this spectral range are based on Holmium-doped fibers and crystals which benefit from their high gain and broad spectral bandwidth to support the generation of ultrashort pulses. ${ }^{1}$ Resonant pumping of $\mathrm{Ho}^{3+}$ lasers leads to a small quantum defect which reduces thermal load and at the same time enhances the overall efficiency of the laser system. In fact, several mJ-level ultrashort pulse amplifiers in this spectral region have been demonstrated mainly utilizing chirped pulse amplification $(\mathrm{CPA})$ in regenerative amplifiers $(\mathrm{RA})^{4,5}$ or cryogenically cooled high-power linear amplifiers. ${ }^{6}$ However, these amplifier systems lack compactness and simplicity for a future step towards a commercial product. An alternative are CPA-free linear amplifiers working at room temperature. In order to boost the pulse energy from fiber-based seed sources at nJ-level to the $\mu J$-level multiple passes through the gain medium, long crystals, high doping concentrations or a combination will be most likely required for proper amplification. In 2012, Dergachev presented a $2 \mu \mathrm{m}$ amplifier with up to $45 \mathrm{~dB}$ gain achieved in a dualcrystal double pass setup with longitudinally pumped Ho(0.5 at.\%):YLF crystals. ${ }^{7}$ This laser amplifier produced ns-pulses with a peak power of up to about $50 \mathrm{~kW}$ at $\mathrm{kHz}$ rate. However, higher peak power at MW-level is preferable regarding nonlinear conversion stages for efficient mid-IR generation.

Here, we present CPA-free linear amplification of 5 ps pulses in Ho:YLF crystals up to $100 \mu \mathrm{J}$ pulse energy at $10 \mathrm{kHz}$ repetition rate maintaining an ultrashort pulse duration of $6.3 \mathrm{ps}$. The seed pulses are provided by a Ho-based all-fiber system consisting of a soliton oscillator and a subsequent pre-amplifier followed by a free-space acousto-optical modulator as pulse-picker. To the best of our knowledge, this is the first $2 \mu \mathrm{m}$ crystal amplifier at $\mu \mathrm{J}$-level extending the $10 \mathrm{kHz}$ repetition rate. In addition, we present first CPA-free amplification in the $2 \mu \mathrm{m}$ wavelength range up to pulse energies of $100 \mu \mathrm{J}$ maintaining ultrashort pulse duration. Considering the achieved pulse peak power at MW-level, this system is a powerful tool for efficient pumping of parametric amplifiers to address the highly demanded mid-IR spectral region with just one nonlinear conversion stage. Furthermore,

Send correspondence to Moritz Hinkelmann

Moritz Hinkelmann: E-mail: m.hinkelmann@lzh.de, Telephone: +49 (0) 511 2788-268

Solid State Lasers XXVII: Technology and Devices, edited by W. Andrew Clarkson, Ramesh K. Shori, Proc. of SPIE Vol. 10511, 1051109 - @ 2018 SPIE · CCC code: 0277-786X/18/\$18 · doi: 10.1117/12.2287387 
this turn-key laser system benefits from a very small footprint and its simplicity compared to complex table-top size CPA RA configurations or cryogenically cooled high-power linear amplifiers in the $2 \mu \mathrm{m}$ spectral region. In detailed numerical simulations we verified our experimental results with theoretical calculations and discuss scaling options for pulse duration and energy.

\section{EXPERIMENTAL SETUP}

The setup of the laser system is outlined in Figure 1 and consists of three main parts, a seed source based on a Ho-doped fiber, an acousto-optic modulator (AOM, AA Opto-Electronic, MT80-A0.4-2000) which serves as the pulse-picker and a linear amplifier based on a Ho:YLF crystal. The seed source is similar to the one previously published ${ }^{8}$ and delivers ultrashort pulses with a pulse duration of $5 \mathrm{ps}$ and a pulse energy of $21 \mathrm{~nJ}$ only limited by the damage threshold of the subsequent free-space AOM. A set of two quarter-wave plates and one half-wave plate is used to adapt the polarization for a proper operation of the AOM. The latter picks the pulses from $24 \mathrm{MHz}$ down to a repetition rate between $1 \mathrm{kHz}$ to $100 \mathrm{kHz}$ with an efficiency of about $55 \%$ into the first diffraction order. The pulse-to-pulse temporal distance of about $42 \mathrm{~ns}$ of the seed laser requires a proper rise and fall time of the AOM that has been achieved by focusing the seed laser beam into the AOM crystal with a beam diameter of about $200 \mu \mathrm{m}$. The input and output beam for the linear amplifier are separated by an optical isolator (Thorlabs, IO-4-2050-HP) with about $90 \%$ transmission at $2050 \mathrm{~nm}$ as specified by the manufacturer. The amplifier is dual-pumped from two sides with $50 \%$ of the pump radiation, respectively, by means of two dichroic mirrors leading to a homogeneous distribution of the inversion level within the amplifying medium. Pump and signal are folded three times through the $20 \mathrm{~mm}$ long Ho:YLF crystal which has an aperture of $5 \times 5 \mathrm{~mm}^{2}$. The Ho:YLF crystal exhibits 1.5 at. $\% \mathrm{Ho}^{3+}$ doping and has anti reflection coatings for pump and signal wavelength on both end surfaces. It is wrapped in Indium foil and mounted on a TEC cooled copper mount which is set to a temperature of $278 \mathrm{~K}$. After the third pass the signal is back reflected such that an overall number of 6 passes contributes to the amplification process.

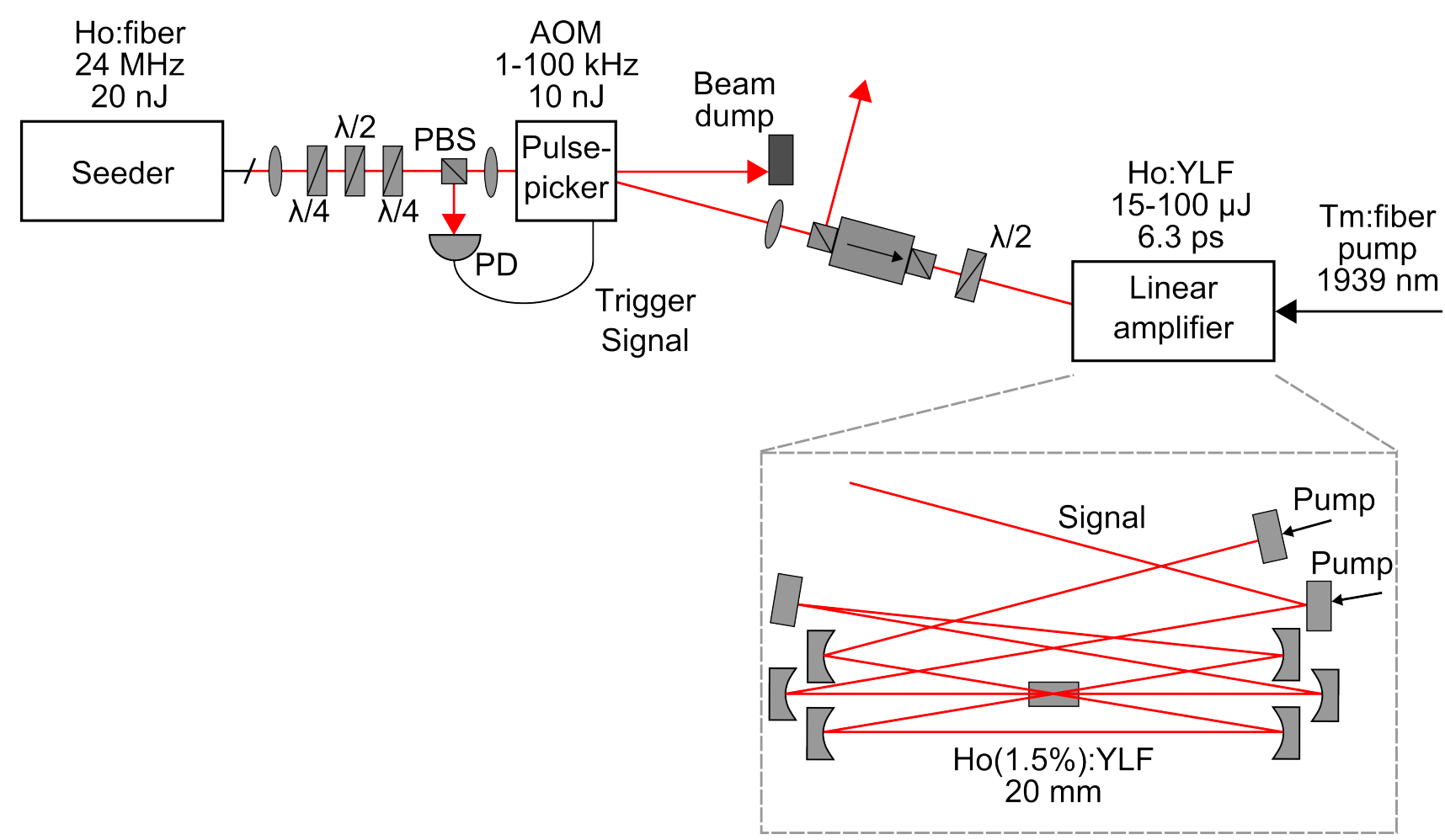

Figure 1. Schematic setup of the amplifier. PD: photo diode, PBS: polarization beam splitter, $\lambda / 2$ : half-wave plate, $\lambda / 4$ : quarter-wave plate.

Up to $10.5 \mathrm{~W}$ of pump power at a wavelength of $1939 \mathrm{~nm}$ is available provided by an in-house built polarization 
maintaining Tm:fiber laser amplifier. Pump and signal polarization can be tuned by half-wave plates to hit the c-axis of the Ho:YLF biaxial crystal for highest amplification efficiency. In order to achieve high saturation, therefore high gain, pump and signal are focused into the crystal by means of curved mirrors. The calculated beam diameter inside the crystal is $250 \mu \mathrm{m}$. We intentionally avoided utilizing lenses due to chromatic aberrations for the pump and signal wavelength that are spectrally separated by $90 \mathrm{~nm}$.

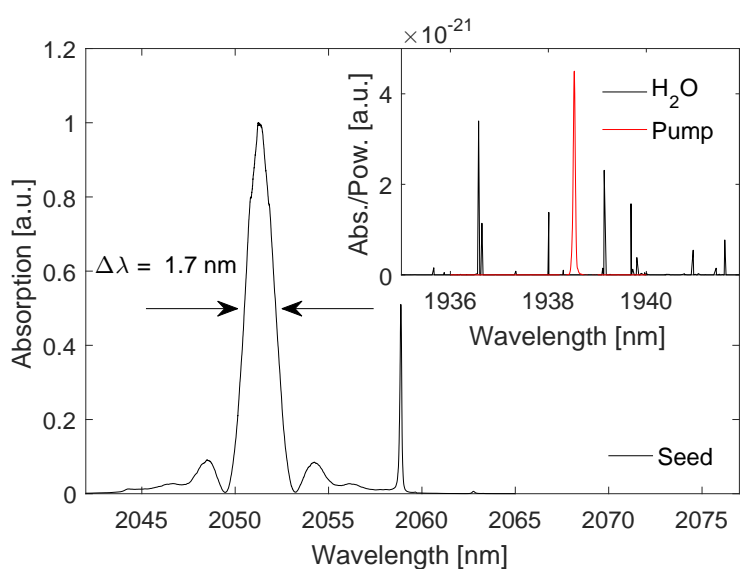

Figure 2. Spectral distribution of the seed pulse. Inset: $\mathrm{H}_{2} \mathrm{O}$ water absorption lines (HITRAN) and spectral distribution of the pump radiation.

This lab-based setup is able to fit a $60 \mathrm{~cm}$ by $90 \mathrm{~cm}$ breadboard which results in a compact package. We believe that further iterative design steps significantly reduce the footprint towards a size that meets the requirements for a commercial product. In order to ensure stable operation and prevent absorption of the pump radiation due to atmospheric water content, we implemented an aluminum housing around the free-space optics of the amplifier which was purged with $\mathrm{N}_{2}$ gas. At the same time, removing the water content of air mitigates condensation of water on the crystal surfaces and, thus, enables proper cooling of the laser crystal. Figure 2 shows the optical spectrum of the used seed pulses and the pump radiation (inset, red line). One can clearly see that the pump emission at $1939 \mathrm{~nm}$ is located between several water absorption lines and purging the housing with $\mathrm{N}_{2}$ gas is mandatory for stable and reliable operation.

\section{NUMERICAL MODEL}

Our numerical simulations are based on the modified Frantz-Nodvik model that has been published by Kroetz et al. in $2016^{9}$ and will be introduced in this section. The wavelength dependent single pass gain in an amplifying medium with length $l$ and dopant ion density $N$ is given as

$$
G(\lambda)=\exp \left(\sigma_{g}(\lambda) N l\right) .
$$

$\sigma_{g}(\lambda)$ represents the spectral gain cross section that is dependent on the characteristic emission and absorption cross section $\sigma_{\text {ems }}(\lambda)$ and $\sigma_{\text {abs }}(\lambda)$ of the gain medium. It can be written

$$
\sigma_{g}(\lambda)=\beta\left(\sigma_{\mathrm{ems}}(\lambda)+\sigma_{\mathrm{abs}}(\lambda)\right)-\sigma_{\mathrm{abs}}(\lambda)
$$

with $\beta$ the inverted fraction as a measure of the population inversion level which can be calculated as follows

$$
\beta=\frac{n_{\mathrm{e}}}{N_{\text {total }}} .
$$

Taking into account an initial fluence $J_{0}(\lambda)$ passing through the gain medium, the amplified or remaining spectral fluence $J_{1}(\lambda)$ can be calculated as 


$$
J_{1}(\lambda)=J_{\mathrm{sat}}(\lambda) T(\lambda) \ln \left(1+G(\lambda)\left(\exp \left(\frac{J_{0}(\lambda)}{J_{\mathrm{sat}}(\lambda)}\right)-1\right)\right)
$$

with the saturation fluence

$$
J_{\mathrm{sat}}(\lambda)=\frac{\mathrm{hc}}{\lambda\left(\sigma_{\mathrm{abs}}(\lambda)+\sigma_{\mathrm{ems}}(\lambda)\right)} .
$$

$T(\lambda)$ in Eq. (4) considers a spectrally dependent transmission loss, $\mathrm{h}$ is Planck's constant and c stands for the speed of light. Here, instead of updating the gain as can be found in the classical expressions, we update the inverted fraction as follows

$$
\beta_{1}=\beta_{0}-\frac{\int\left[\lambda\left(\frac{J_{1}(\lambda)}{T(\lambda)}-J_{0}(\lambda)\right)\right] \mathrm{d} \lambda}{\mathrm{hcl} N} .
$$

Eq. (1)-(6) hold their validity for both the absorption of pump radiation as well as the amplification of an initial seed pulse. However, for $\mathrm{kHz}$ repetition rates, thus, long continuous wave pumping processes, we need to consider a correction term for inversion decay losses. Kroetz et al. added a correction factor that is based on the inversion decay law ${ }^{9}$ with

$$
\beta^{*}=\beta \exp \left(-\frac{\Delta t}{\tau_{\text {gain }}}\right),
$$

where $\tau_{\text {gain }}$ is the upper laser level lifetime of the gain medium and $\Delta t$ represents the duration of the pump interval. Next to dividing the pump and seed fluences temporally into slices and consecutively simulating the inverted fraction after each slice ${ }^{9}$ to simulate homogeneous broadening, we split our gain medium in slices with $1 \mathrm{~mm}$ thickness. The inverted fraction is solved for every crystal slice independently which accounts for an inhomogeneous distribution of absorbed pump power within the gain medium. We also adapted the simulation such that it considers the pumping from two directions as well as folding the beam several times through the crystal.

\section{EXPERIMENTAL RESULTS}
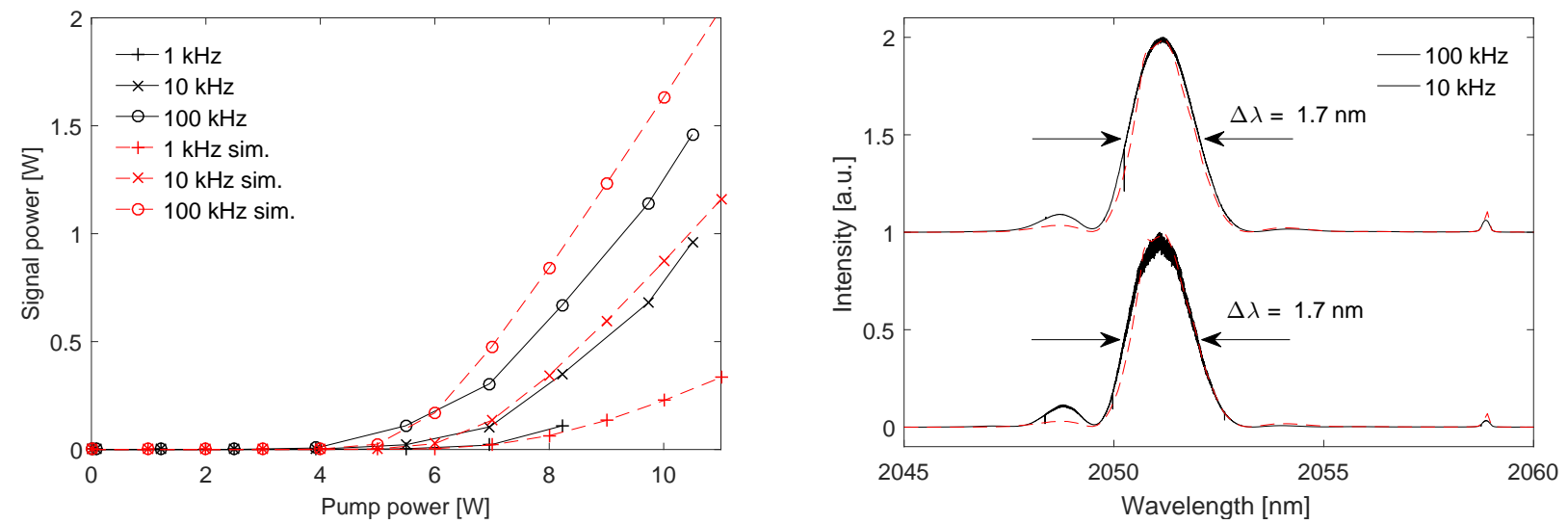

Figure 3. Left: Measured optical output power with increasing pump power (black solid line) and corresponding simulations (red dashed line) for different repetition rates. Right: Measured optical output spectrum (black solid line) for $100 \mathrm{kHz}$ and $10 \mathrm{kHz}$ repetition rate and corresponding simulations (red dashed line). 
By using a seed pulse energy of $9 \mathrm{~nJ}$ and a pulse duration of $5 \mathrm{ps}$ we measured the output power of the amplifier versus increasing pump power for different repetition rates. The achieved experimental results as well as the numerical results are shown in Figure 3 left. The simulation results take into account the spectral distribution of the seed and pump radiation as well as losses, beam sizes, operation parameters and Ho:YLF crystal properties such as fluorescence lifetime $\tau_{\mathrm{f}}=14 \mathrm{~ms}^{10}$ or the emission and absorption cross section $\sigma_{\text {ems }}$ and $\sigma_{\text {abs }}$ for $E \|$ c. ${ }^{11}$ Due to damage within the crystal for pulse energies above $100 \mu \mathrm{J}$ we did not increase the pump power at $1 \mathrm{kHz}$ repetition rate. However, pulse energies above $100 \mu \mathrm{J}$ seem to be feasible because no saturation in the three curves can be observed. In addition, Figure 3 presents the optical spectrum for the $100 \mu \mathrm{J}$ pulses at $10 \mathrm{kHz}$ and for $14 \mu \mathrm{J}$ pulse energy at $100 \mathrm{kHz}$ repetition rate. The corresponding Fourier-limited pulse duration is $2.3 \mathrm{ps}$ and could potentially be achieved with proper compression following the linear amplifier stage of our laser system. However, such compression stages based on e.g. reflection gratings lack compactness and cause severe losses reducing the available pulse energy.

The experimental results coincide with the numerical simulations for both the pulse energy and the spectral distribution. This proves the validity of our model and allows to discuss scaling options for the pulse duration and energy of this laser setup. In our simulations increasing the pump power is straight forward and the numerical results predict pulse energies up to the mJ-level without increasing the amount of passes through the Ho:YLF crystal. However, as mentioned above, one has to take into account the damage threshold of the gain medium. This can be overcome by increasing the spot size within the Ho:YLF crystal or a sequential design of two linear amplifiers. Since in our setup the first pass naturally has the same beam diameter as the last pass, a two crystal setup benefits from the ability to adapt the beam size to the pulse energy such that the first pass can have a small diameter for high saturation, thus, high gain whereas the last pass has a larger focal beam waist. It has to be noted that the combination of high doping concentrations above 1.0 at.\% and high photon flux may lead to the onset of upconversion processes ${ }^{1}$ which limit the the achievable gain per pass in Ho:YLF.

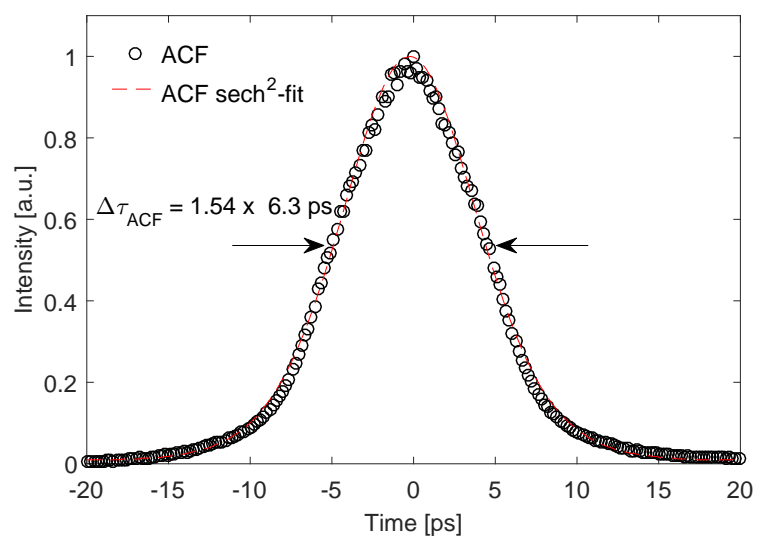

Figure 4. Measured autocorrelation trace for a pulse energy of $100 \mu \mathrm{J}$ at $10 \mathrm{kHz}$ repetition rate.

Figure 4 presents the measured autocorrelation trace of pulses with an output energy of $100 \mu \mathrm{J}$ at $10 \mathrm{kHz}$ repetition rate. The pulse duration can be estimated to $6.3 \mathrm{ps}$, assuming a squared hyperbolic secant shape $\left(\operatorname{sech}^{2}\right)$. Due to some amount of gain narrowing in combination with nonlinear effects the pulse duration is slightly longer than the input pulse duration. Nevertheless, this duration still belongs to the ultrashort pulse range. Taking into account the pulse energy of $100 \mu \mathrm{J}$, we achieved a pulse peak power of about $13.9 \mathrm{MW}$. Our simulations show that Ho:YLF is capable of producing a bandwidth of $2.6 \mathrm{~nm}$ and a corresponding Fourier-limited pulse duration around 1.6 ps. Sub-ps pulses can be achieved when taking advantage of the full M-shaped gain bandwidth including the emission peak around $2063 \mathrm{~nm}$. Though proper spectral shaping of the input seed will be essential for broadest output spectra in order to obtain a Gaussian-shaped rather than a M-shaped output spectrum. 


\section{CONCLUSION}

In conclusion, we demonstrated a CPA-free multi-pass linear amplification in Ho:YLF up to $100 \mu \mathrm{J}$ maintaining ultrashort pulse duration of $6.3 \mathrm{ps}$ at $10 \mathrm{kHz}$ repetition rate. These values correspond to a pulse peak power of $13.9 \mathrm{MW}$ making this laser a potential candidate for subsequent mid-IR generation in parametric conversion stages based on e.g. ZGP. Numerical simulations verify our experimental results and show scaling options considering higher pulse energy towards the mJ-level.

\section{ACKNOWLEDGMENTS}

This work is funded by the German Federal Ministry of Education and Research (BMBF) (13N13974 NUKLEUS).

\section{REFERENCES}

[1] Scholle, K., Lamrini, S., Koopmann, P., and Fuhrberg, P., "2 $\mu \mathrm{m}$ laser sources and their possible applications," in Frontiers in Guided Wave Optics and Optoelectronics , 471-500. B. Pal, ed. (InTech, 2010).

[2] Leindecker, N., Marandi, A., Byer, R. L., Vodopyanov, K. L., Jiang, J., Hartl, I., Fermann, M., and Schunemann, P. G., "Octave-spanning ultrafast OPO with 2.6-6.1 $\mu \mathrm{m}$ instantaneous bandwidth pumped by femtosecond Tm-fiber laser," Opt. Express 20(7), 7046-7053 (2012).

[3] Vodopyanov, K. L., "Parametric generation of tunable infrared radiation in $\mathrm{ZnGeP}_{2}$ and GaSe," J. Opt. Soc. Am. B 10(9), 1723-1729 (1993).

[4] Wienke, A., Wandt, D., Morgner, U., Neumann, J., and Kracht, D., "Comparison between Tm:YAP and Ho:YAG ultrashort pulse regenerative amplification," Opt. Express 24(8), 8632-8640 (2015).

[5] L. von Grafenstein, M. Bock, D. U. U. G. and Elsaesser, T., "Ho:YLF chirped pulse amplification at kilohertz repetition rates - 4.3 ps pulses at $2 \mu \mathrm{m}$ with GW peak power," Opt. Letters 41(20), 4668-4671 (2016).

[6] Hemmer, M., Sanchez, D., Jelinek, M., Smirnov, V., Jelinkova, H., Kubecek, V., and Biegert, J., "2-um wavelength, high-energy Ho:YLF chirped-pulse amplifier for mid-infrared OPCPA," Opt. Letters 40(4), 451-454 (2015).

[7] Dergachev, A., "High-energy, kHz-rate, picosecond, 2- $\mu \mathrm{m}$ laser pump source for mid-IR nonlinear optical devices," Proc. SPIE , 85990B (2013).

[8] Hinkelmann, M., Wandt, D., Morgner, U., Neumann, J., and Kracht, D., "Mode-locked Ho-doped laser with subsequent diode-pumped amplifier in an all-fiber design operating at $2052 \mathrm{~nm}$," Opt. Express 25(17), 20522-20529 (2017).

[9] Kroetz, P., Ruehl, A., Murari, K., Cankaya, H., Kärtner, F. X., Hartl, I., and Miller, R. J. D., "Numerical study of spectral shaping in high energy Ho:YLF amplifiers," Opt. Express 24(9), 9905-9921 (2016).

[10] Payne, S. A., Chase, L. L., Smith, L. K., Kway, W. L., and Krupke, W. F., "Infrared cross-section measurements for crystals doped with $\mathrm{Er}^{3+}, \mathrm{Tm}^{3+}$, and $\mathrm{Ho}^{3+}$," IEEE J. Quantum Electron. 28(11), 2619-2630 (1992).

[11] Walsh, B. M., Barnes, N. P., and Bartolo, B. D., "Branching ratios, cross sections, and radiative lifetimes of rare earth ions in solids: Application to $\mathrm{Tm}^{3+}$ and $\mathrm{Ho}^{3+}$ ions in $\mathrm{LiYF}_{4}$," J. of Appl. Phys. 83(5), 2772-2787 (1998). 